\title{
Multi-channel DTV signal generator design using modified Farrow filter
}

\author{
Seo Weon $\mathrm{Heo}^{1}$ and Hyungsuk Kim ${ }^{2 \mathrm{a})}$ \\ ${ }^{1}$ Electronic and Electrical Engineering, Hongik University \\ 72-1 Sangsu-Dong, Mapo-Gu, Seoul, 121-791, Korea \\ ${ }^{2}$ Electrical Engineering, Kwangwoon University \\ 20 Wolgye 1-Dong, Nowon-Gu, Seoul, 139-701, Korea \\ a) hskim@kw.ac.kr
}

\begin{abstract}
This paper proposes a compact multi-channel DTV signal generator. To combine multi-channel signal sources in digital domain, the baseband signal is up-sampled and interpolated using the modified Farrow-type filter. We formulate the optimization problem and design the filter coefficients. The designed filter contains a small number of multipliers with more than $40 \mathrm{~dB}$ sideband signal suppression capability.
\end{abstract}

Keywords: DTV, interpolation filter, Farrow structure, multichannel

Classification: Wireless communication hardware

\section{References}

[1] Advanced Television System Committee, "ATSC mobile DTV standard, part2 - RF/transmission system characteristics," Doc. A/153, Oct. 15, 2009.

[2] H. N. Kim, Y. T. Lee, and S. W. Kim, "Mathematical modeling of VSBbased digital television systems," ETRI J., vol. 25, no. 1, pp. 9-18, Feb. 2003.

[3] R. A. Losada and R. Lyons, "Reducing CIC filter complexity," IEEE Signal Process. Mag., vol. 23, no. 4, pp. 124-126, July 2006.

[4] J. T. Kim, "Efficient polynomial interpolation filters with symmetric coefficients," IEEE Vehicular Technology Conference, vol. 5, pp. 2276-2279, May 2006.

[5] C. W. Farrow, "A continuously variable digital delay element," Proc. IEEE ISOCS, vol. 3, pp. 2641-2645, June 1988.

[6] J. O. Smith and P. R. Cook, "The second-order digital waveguide oscillator," Proc. ICMC, pp. 150-153, 1992.

\section{Introduction}

DTV technology has been constantly evolving, including recent mobile applications, such as the ATSC 8-VSB [1], DVB-T2 and ISDB-T. We need signal sources that can generate DTV signals in real time to test the receivers 
for these various communication technologies. Recently developed DTV receivers usually have the capability of channel browsing, where several channel output streams are displayed simultaneously so that users can easily choose the channel to watch. To test or show demonstrations of this multi-channel browsing TV receiver, conventionally we usually deploy several equipments of single-channel DTV transmitter and combine them with an RF combiner but that is both too bulky and expensive solution. In this paper, we propose a compact multi-channel signal generator where several DTV channel signals [2] are generated, combined in digital domain and up-converted to the desired RF channel directly.

We need to increase the sampling rate to reserve spaces in the frequency domain for up-conversion and channel combination for generating multichannel signals in the digital domain. Several interpolation filter architectures, such as the multi-stage band-pass filter, cascaded integrator and comb (CIC) filter [3], or wave digital filter can be applied. In this paper, we design a modified Farrow type interpolation filter $[4,5]$ for the proposed system and show that the designed filter shows good sideband suppression capability with just a small number of multipliers.

\section{Proposed multi-channel DTV signal generator}

Fig. 1 (a) shows the block diagram of the proposed multi-channel DTV signal generator. To reduce the system complexity, we design the system in part by SW and the other part by HW module. In this paper, we explain the system based on the ATSC DTV technology [1] but the design can be applied to any of the DTV technology.

First, MPEG TP (transport packet) or IP packet data are processed by the SW module, which includes the symbol rate processing block such as the scrambler, Reed-Solomon encoder, symbol interleaver and TCM encoder. The output data processed by the SW module are transferred to the HW module via the USB 2.0 connection. The HW module processes the waveform shaping filtering (square root raised cosine filter, SRRC), interpolation filtering to increase the sampling rate to reserve spaces in the frequency domain for multi-channel combining. Each channel signal is up-converted to the adjacent low frequency IF channels and combined digitally. To consider the applications where we have no access to the TP/IP signal sources such as the demo booth or the production lines, we add the NAND/DDR2 memory to store the SW processed data stream transferred by the USB 2.0 connection. In this case, the stored data stream inputs to the HW module repeatedly.

Fig. 1 (b) shows the HW module operations in the frequency domain. First, the data processed by the SW module are mapped to the 8 level VSB signal with pilot insertion. Since ATSC DTV system use real signaling scheme (, i.e., not quadrature signaling), the system cuts off the left half part of the frequency spectrum as is shown in Fig. 1(b2) using the complex waveform shaping filter. The complex waveform shaping filter is composed of square root raised cosine (SRRC) filter and Hilbert transformed SRRC filter 


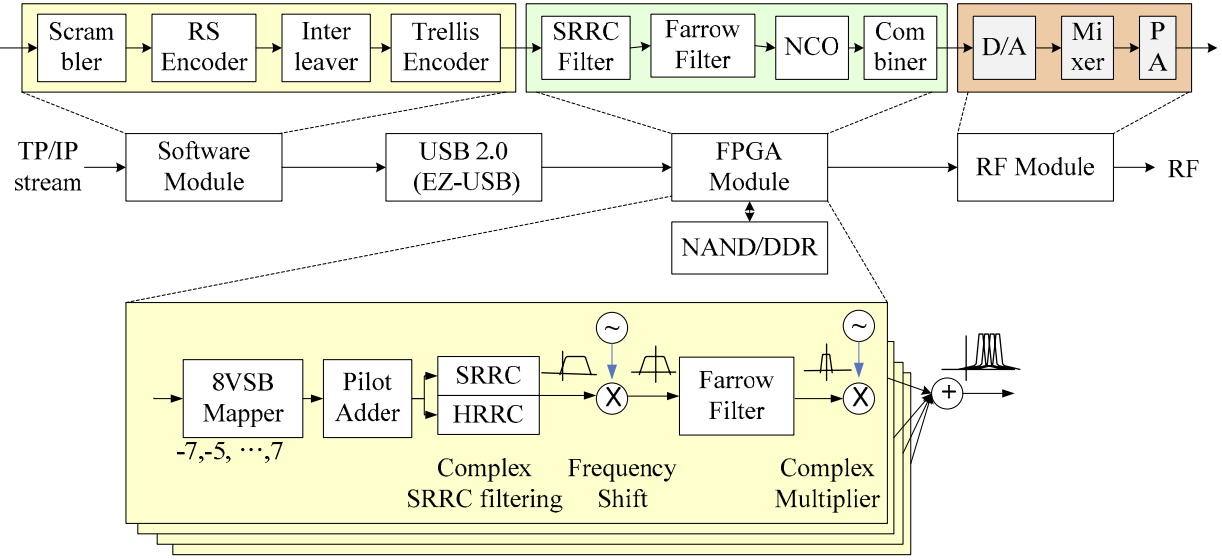

(a)

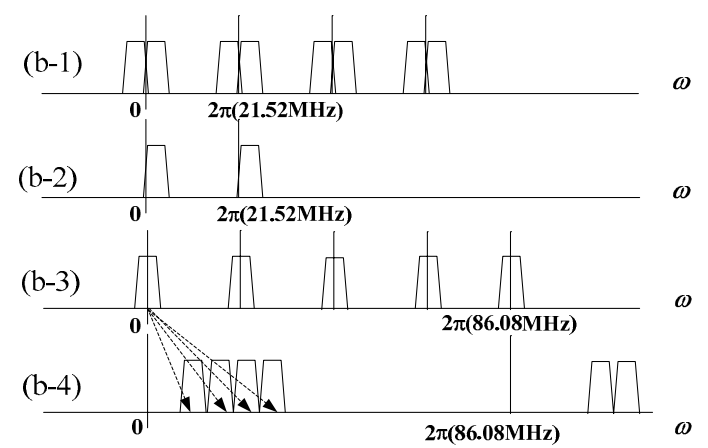

(b)

Fig. 1. (a) The proposed system block diagram, (b) system operation in frequency domain.

(HRRC).

After shifting the center frequency to aid the subsequent interpolation process, the system increases the sampling rate by 4 as is shown in Fig. 1 (b3). The interpolation filter processes the zero inserted signals to remove the image signals. Among the several candidates of interpolation filter technologies, a modified Farrow filter was adopted to reduce the hardware complexity, i.e., the number of multipliers and adders. After reserving the spaces in the frequency domain by interpolation process, the system up-converts each channel signal by multiplying them with the complex exponential carrier signal generated by the digital waveguide oscillator [6]. Finally, those signals are added together in the digital domain to form the multi-channel signal. The system converts the digitally combined signal to analog signal by DAC and processes further to generate the final RF waveform.

\section{Design of Farrow interpolation filter}

As explained in the previous Section, we need interpolation filter for multichannel combining in the digital domain. We adopt the Farrow structure to reduce the hardware complexity of the interpolation filtering by the fact that the number of the required multipliers is small compared with the other filter structures and the filter length does not increase significantly as the up-sampling ratio increases. Fig. 2 shows the Farrow filter composed of three 


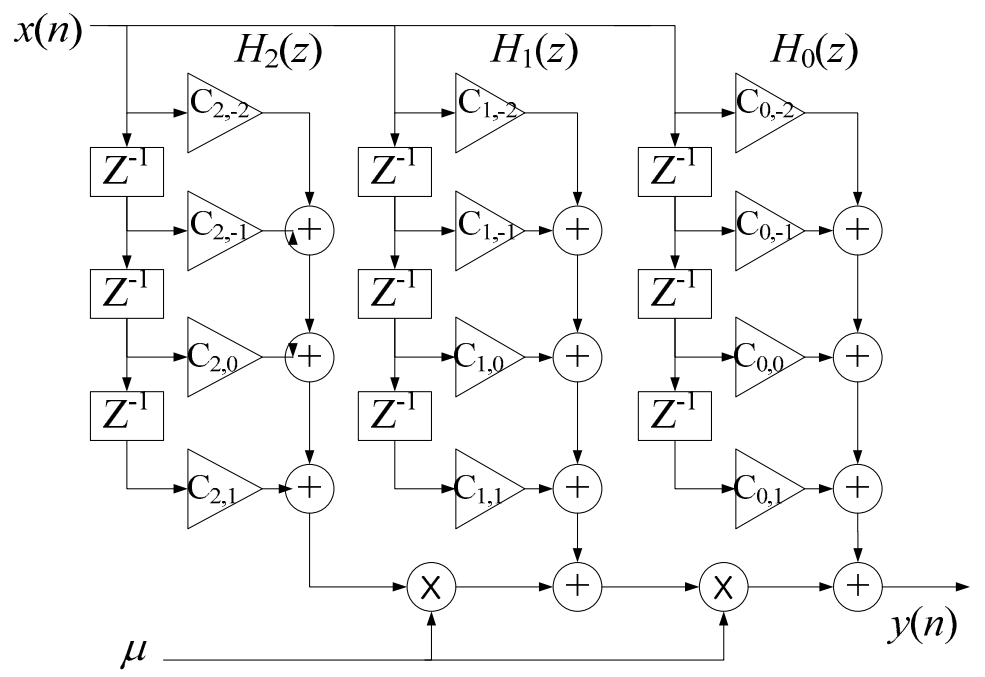

Fig. 2. Farrow filter block diagram.

sub filters $\left(H_{i}(z), i=0,1,2\right)$ whose length is 4 . Several modified Farrow structures have been proposed exploiting the symmetry properties of the impulse response to reduce the number of multipliers. We adopt the modified Farrow filter proposed in [5] which requires the least number of multipliers among those filters known so far, but unfortunately no design process or results are shown in the literature. So we formulate the optimization problems that are appropriate for our applications in the followings.

\subsection{Mathematical expression of the Farrow filter}

Farrow interpolator filter can be described mathematically as

$$
\begin{aligned}
H(\mu, z) & =\sum_{m=0}^{M} \mu^{m} H_{m}(z)=\sum_{m=0}^{M} \mu^{m} \sum_{l=-L}^{L-1} c_{m, l} z^{l} \\
H(z) & =\sum_{\mu=0}^{K-1} z^{-\mu} H\left(\mu / K, z^{K}\right)=\sum_{\mu=0}^{K-1} \sum_{m=0}^{M}(\mu / K)^{m} z^{-\mu} H_{m}\left(z^{K}\right) \\
& =\sum_{\mu=0}^{K-1} \sum_{m=0}^{M}(\mu / K)^{m} z^{-\mu} \sum_{l=-L}^{L-1} c_{m, l} z^{-K l}
\end{aligned}
$$

where $M$ is an order of the approximating polynomial, $2 L$ is the number of taps of each sub-filters, $K$ is the up-sampling ratio, $c_{m, l}$ is the coefficient of the $l^{\text {th }}$ tap of the $m^{\text {th }}$ sub-filter. $H(z)$ is the overall filter and $H(\mu, z)$ is the $\mu^{\text {th }}$ phase sub-filter.

If we denote the above equations in matrix form, the $\mu^{\text {th }}$ phase sub-filter $H(\mu, z)$ can be described mathematically as

$$
H(\mu, z)=\sum_{m=0}^{M} \mu^{m} H_{m}(z)=\sum_{m=0}^{M} \sum_{l=-L}^{L-1} \mu^{m} c_{m, l} z^{l}=b_{\mu}^{T} C Z,
$$

where

$$
b_{\mu}^{T}=\left[\begin{array}{llll}
1 & \mu & \ldots & \mu^{M}
\end{array}\right], Z^{T}=\left[\begin{array}{llll}
z^{-L} & z^{-L+1} & \ldots & z^{L-1}
\end{array}\right],
$$




$$
C=\left[\begin{array}{cccc}
c_{0,-L} & c_{0,-L+1} & \ldots & c_{0, L-1} \\
\vdots & & & \\
c_{M,-L} & c_{M,-L+1} & \ldots & c_{M, L-1}
\end{array}\right] .
$$

Using the matrix identity of $A V B^{T}=(A \otimes B) \operatorname{vec}(V), H(\mu, z)$ can be described by

$$
\begin{aligned}
& H(\mu, z)=A(\mu, z) X= \\
& \quad\left[z^{-L} \ldots z^{L-1} \mu z^{-L} \ldots\right]\left[c_{0,-L} \ldots c_{0, L-1}, \ldots, c_{M,-L}, \ldots, c_{M, L-1}\right]^{T},
\end{aligned}
$$

where $\mathrm{A}(\mu, z)=b_{\mu}^{T} \otimes Z^{T}$ and $X=\operatorname{vec}(C)$. Then the overall interpolation filter can be described by

$$
H(z)=\sum_{\mu=0}^{K-1} z^{\mu} A\left(\mu / K, z^{K}\right) X=B(z) X .
$$

\subsection{Optimization of filter coefficients}

The interpolation filter should be designed such that the image signal due to the interpolation process is suppressed well below the desired signal power. In the proposed multi-channel modulation system where we consider $N$-channel combining in the digital domain, the filter should suppress $N$-1 image signals in the frequency domain. Based on the convex optimization theory, the optimization problem can be summarized by the least square criterion of

$$
\underset{X}{\arg \min } \int_{-\pi}^{\pi}\left|B\left(e^{j \omega}\right) X-H_{d}\left(e^{j \omega}\right)\right|^{2} d \omega=\underset{X}{\arg \min }\left\{X^{T} Q X-2 P X\right\},
$$

where $H_{d}\left(e^{j \omega}\right)$ is the desired frequency response and

$$
Q=\int_{-\pi}^{\pi} B^{H}\left(e^{j \omega}\right) B\left(e^{j \omega}\right) d \omega, P=\int_{-\pi}^{\pi} \operatorname{Re}\left\{H_{d}\left(e^{j \omega}\right) B^{H}\left(e^{j \omega}\right)\right\} d \omega .
$$

The least square optimization result shows that the image signal attenuation level is not uniform in the frequency domain. To gain uniform stop-band suppression capability we can also apply the minimization of maximum error (min-max) criterion in the stop-band, which is given by

$$
\begin{aligned}
& \underset{X}{\arg \min }\left\{\max _{\omega}\left|B\left(e^{j \omega}\right)-H_{d}\left(e^{j \omega}\right)\right|\right\} \approx \\
& \underset{X}{\arg \min }\left\{\max _{\omega \in\left\{\omega_{i}, i=1,2, \ldots, N_{f}\right\}}\left|B\left(e^{j \omega}\right)-H_{d}\left(e^{j \omega}\right)\right|\right\},
\end{aligned}
$$

where $\omega_{i}$ is the $i^{\text {th }}$ sampling (grid) frequency to make the continuous time function optimization to discrete time function optimization problem. The Matlab function of quadprog or fminmax can be applied to solve these optimization problems.

\section{Design results}

Fig. 3 (a) shows the designed modified Farrow filter structure where we assumed $3^{\text {rd }}$ order polynomial approximation and filter length of $4(M=3$, 

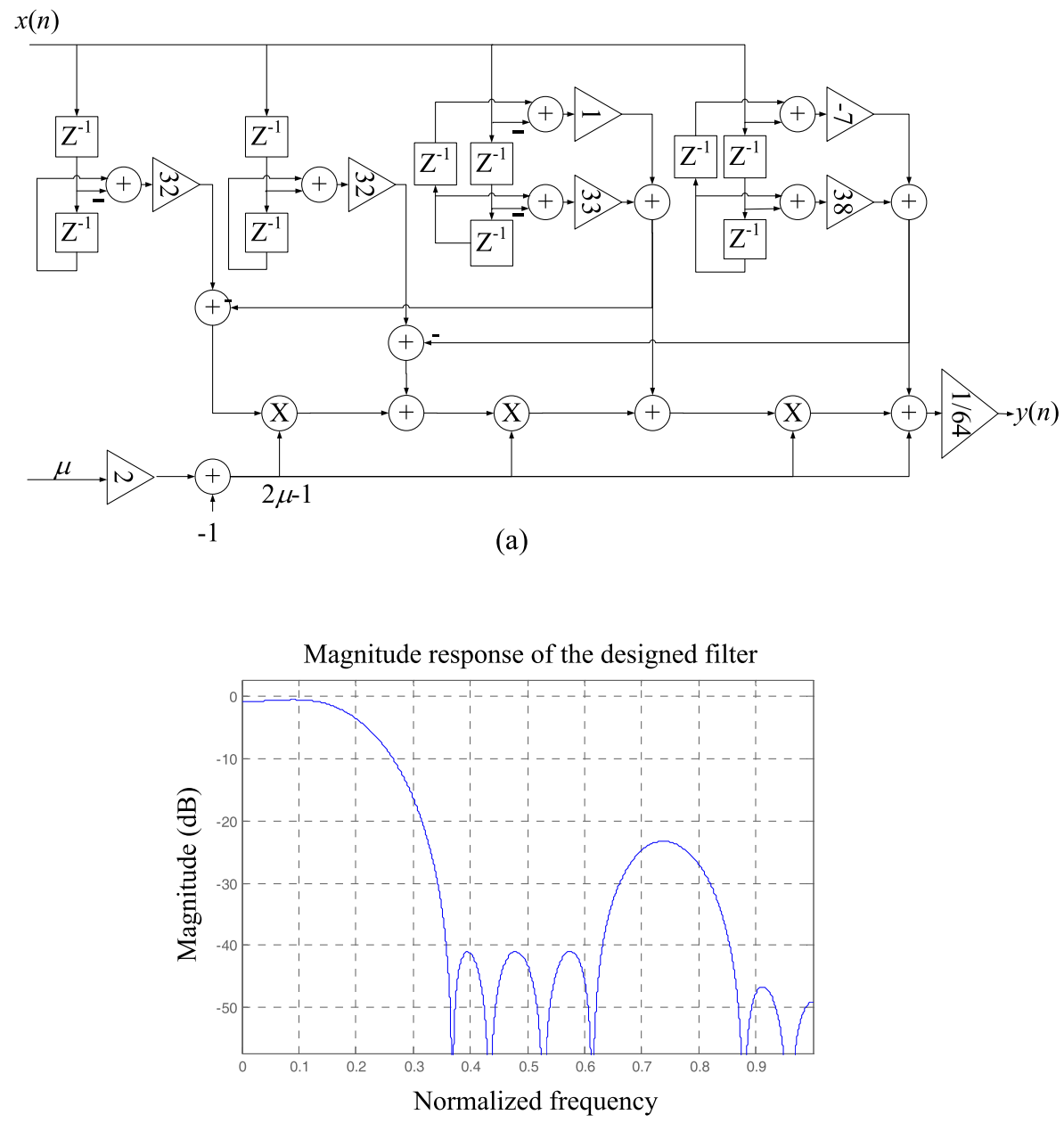

(b)

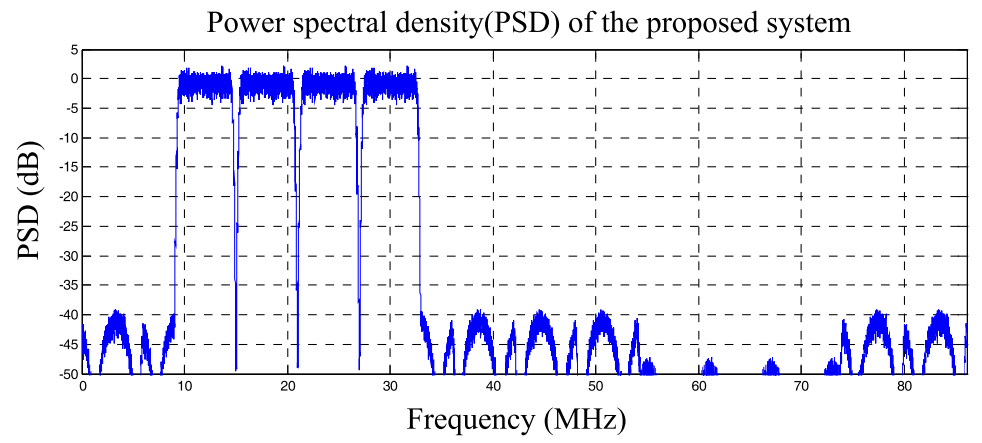

(c)

Fig. 3. (a). Designed Filter, (b) Filter magnitude response, (c). PSD of combined signal.

$L=2$ ) with 8 bit coefficient quantization. It requires only three simple integer multiplications with just a small number of additional shifters and adders. To design the filter with multi-stage LPF requires more than 20 tap filters and CIC filter require an 11 tap CIC compensation filter in addition to the several stage high precision accumulators when designed using the Matlab filter design toolbox. Reducing the number of multipliers is important to fit into the proper size FPGA. 
Fig. 3 (b) shows the frequency response of the designed filter based on the min-max criterion. With just a small number of filter taps and polynomial order, we could get more than $40 \mathrm{~dB}$ image signal suppression capability (see between $0.4 \sim 0.6$ in the normalized frequency where the image signal exists in the magnitude response graph).

The power spectral density (PSD) of the four-channel combined signals is shown in Fig. 3 (c) after the whole processing is executed by the Matlab fixed point simulator. The result shows that our proposed system generates multichannel VSB signal with almost $40 \mathrm{~dB}$ sideband suppression capability. To confirm the performance, we demodulated the transmitted signal in Matlab using a reference demodulator which has very sharp band-pass shaping filter and assumes perfect timing/carrier recovery. We could get more than $38 \mathrm{~dB}$ SNR for any of the four channel signals. The whole system was implemented and verified using the Xilinx Virtex-4 LX-200 FPGA (with almost $90 \%$ of the DSP48 usage), PC SW, Cypress CY7C68013 EZ-USB device and analog modules.

\section{Conclusion}

This paper proposes a multi-channel DTV signal generator. The system is composed of several sub-systems operating partly by SW and partly by HW module. The multi-channel MPEG TP/IP streams processed by the SW module are transferred HW module via the USB 2.0 connection. The system deploys a modified Farrow structure for data interpolation to combine multichannel signals digitally. We formulate two optimization problems based on the least square and min-max error criterion and design the filter coefficients. The results show that the designed filter has more than $40 \mathrm{~dB}$ image signal suppression capability with a small number of multipliers.

\section{Acknowledgments}

This work was supported by the research grant of Basic Science Research Program through the National Research Foundation of Korea (NRF) funded by the Ministry of Education, Science and Technology (2011-0007066), Kwangwoon University 2011. 